\title{
New Divisions and Group
}

\section{High Energy and Elementary Particle Physics Division}

The formation of a High Energy and Particle Division was approved by the Council at its meeting in Paris. The members of the Steering Committee which proposed the Division were asked to form an Interim Board to bring the Division into operation. Its Board is composed of A. Berthelot, R.H. Dalitz, W. Paul, P. Preiswerk and A. Zichichi. A.M. Baldin was also approved as a member of the Committee during the Council Session.

The Council also agreed the broad terms of the functions and aims formulated by the Steering Committee at its meeting in Geneva on 5 February. These are as follows:

1. The Division should serve as a forum for discussion of professional problems in high energy physics, and should endeavour to express general opinions, and to take appropriate action, based on the views of the physicists working in the field.

2. Concerning the general aims and activities of the Division, the Steering Committee proposes:

a) to establish a body within the Division for the purpose of furthering co-ordination in the development and use of the research facilities in all aspects of high energy physics in Europe;

b) to improve the organization and co-ordination of conferences in this field in Europe, and in particular to promote small conferences on specialized topics;

c) to further the co-ordination of advanced education in high energy physics and related fields, and to promote new activities where needed.

\section{Nuclear Physics Division}

The formation of a Nuclear Physics Division was approved by the Council at its meeting in Paris. The proposal to form such a Division was first dis- cussed at the Conference on Heavy Ion Physics held in Heidelberg in July 1969. An Interim Committee to bring the Division into operation is composed of L.L. Green (Chairman), N. Cindro and R. van Lieshout (Regional Secretaries), E. Cotton, W. Gentner, H. Schmidt-Rohr, G.H. Stafford and V.V. Volkov.

The Division aims to foster European collaboration in the field of nuclear physics and looks forward also to close collaboration with the High Energy and Particle Physics Division. The Division intends to be active in the field of rationalization and coordination of conferences and summerschools, to encourage personnel exchange at all levels between nuclear physics research centres, and to be active in public relations and popularization on behalf of nuclear physics.

\section{Computational Physics Group}

Computation is an established feature of research in many branches of physics to-day. To cite but a few, high energy physics, plasma physics, nuclear physics, astrophysics and geophysics make extensive use of computers in some aspects of experimental or theoretical research. With the increasing availability of computational facilities and the falling costsper-operation resulting from new technologies, we may expect during this decade to see both a marked increase in the amount of computation done in physics research and the application of computational techniques to more branches of physics.

There is a very broad range of the kinds of use made of computers in physics. They are used to solve complex sets of equations, to handle large quantities of data, to simulate physical situations and devices or to control experimental equipment recording and processing data in real time. Advances in computer technology and programming may equally bring new ways of applying computers and open up new avenues of research which would otherwise not be feasible.
In such a situation, when a rapidly developing technique is finding applications in many different branches of physics, contacts and exchanges between physicists using these tools will be of great value in stimulating new approaches to problems and shaping the development of the techniques to meet physicists needs. The European Physical Society could, in a unique way, foster such contacts and exchanges, and a proposal to this end was made to the recent meeting of the EPS Council in Paris.

The Council approved the formation of an Inter-Divisional Group to be called the Computational Physics Group. An Interim Committee which will bring the Group into operation, organize its initial programme and establish the necessary links with the various Specialized Divisions, is composed of J.B. Adams, H. Bross, K. Differt, M.R. Feix, B.H. Flowers, E. Knighting, G.R. Macleod (Chairman), B. McNamara (Secretary), C. Moser, R.S. Pease, C.L. Pekeris, K.V. Roberts, A. Vaciago, L. van Hove and L. Verlet

The scope of computational physics covers all aspects of the application of computers to physics research. It is therefore concerned with the formulation of physics problems for computation, with computational and numerical methods as well as with some aspects of computer science such as programming languages, computer system design, graphical computing etc.

The aims of the Computational Physics Group are to provide a forum where physicists active in different branches of physics can discuss common problems in computational physics, exchange ideas and computer programs and foster European collaboration in the development of computational methods and tools in the context of physics research. To meet these aims, the Group will be active in promoting the organization of conferences, specialist meetings, schools, etc. in this field in Europe, and will promote other activities where appropriate. 